PREFERENCIAS DE CONSUMIDORES DE SALSAS PICANTES GUASALZAS DE COMALCALCO, TABASCO.

\title{
PREFERENCIAS DE CONSUMIDORES DE SALSAS PICANTES GUASALZAS DE COMALCALCO, TABASCO
}

\section{GUASALZAS HOT SAUCE CONSUMERS' PREFERENCE IN COMALCALCO, TABASCO}

\author{
Vanesa Sánchez Mejía*, Griselda Márquez Alcudia**, \\ Carlos Alberto Paz Gómez ${ }^{\star \star *}$
}

* Egresada de la Licenciatura en Administración. Universidad Juárez Autónoma de Tabasco. ORCID: https://orcid.org/0000-0002-0851-5581.

**Licenciada en Relaciones Comerciales. Universidad Politécnica del Golfo de México. Email: grisimaral@hotmail.com. ORCID: https://orcid.org/0000-0003-2827-8090.

***Maestro en Administración. Profesor Investigador de la División Académica de Ciencias Económico Administrativas. Universidad Juárez Autónoma de Tabasco. Email: pazgomez.carlosalberto09@gmail.com. ORCID: https://orcid.org/0000-0002-4610-0512.

Dirección para correspondencia: sanchezmejia94@gmail.com 
PREFERENCIAS DE CONSUMIDORES DE SALSAS PICANTES GUASALZAS DE COMALCALCO, TABASCO.

\section{RESUMEN}

OBJETIVO: Determinar las preferencias de los consumidores de salsas picantes de Comalcalco, Tabasco, así como de los principales ingredientes en la cultura gastronómica en otras ramas y datos estadísticos de su producción.

MATERIAL Y MÉTODO: El presente estudio, tuvo un enfoque cuantitativo. Se implementó la técnica de cuestionarios que fueron aplicados a una muestra por conveniencia de 80 personas entre las que destacaron clientes de la misma empresa, así como amistades y familiares.

RESULTADOS: Con base en la información se seleccionaron personas que habían consumido salsas Guasalzas. La muestra fue de 61\% mujeres y 39\% hombres. Se observó que las mujeres demostraron más interés en el consumo de las salsas. También se descubrió la preferencia del chile habanero que fue del $46 \%$ y sólo el $23 \%$ prefirieron el chile amashito.

CONCLUSIONES: El chile es un cultivo originario de México muy importante a nivel mundial. Los usos múltiples del chile y sus derivados datan desde la época prehispánica y van más allá de conformar un extraordinario condimento. En cuanto a la preferencia de los consumidores, se concluyó que las Salsas Guasalzas tienen un alto porcentaje de posibilidades de ser consumidas en Comalcalco, Tabasco.

PALABRAS CLAVE: Chiles. Preferencias de consumidores. Gastronomía.

\section{ABSTRACT}

OBJECTIVE: To determine hot sauce consumers' preferences in Comalcalco, Tabasco. As well as the main ingredients in the local cuisine in other areas and in statistical data in its production.

MATERIAL AND METHOD: For this quantitative study, a questionnaire was administered to 80 people by convenience among clients, friends and family members.

RESULTS: Based on the information, a group of people, who have already tasted the sauce, was selected. The sample was formed by $61 \%$ women and $39 \%$ men. A preference was observed of $46 \%$ on habanero chile and only a $23 \%$ on amashito chile. 
PREFERENCIAS DE CONSUMIDORES DE SALSAS PICANTES GUASALZAS DE COMALCALCO, TABASCO.

CONCLUSIONS: The Mexican origin and the growth of chile is very important worldwide. The multiple uses of chile and its similarities have origin in the Prehispanic epoch and it is considered as an extraordinary ingredient. As for the consumers' preference, it is concluded that Guasalzas Sauce has a high possibility of being consumed in Comalcalco, Tabasco.

KEY WORDS: Chiles. Consumer's preferences. Cuisine.

\section{INTRODUCCIÓN}

México es el país con la mayor diversidad de Capsicum annuum, donde se cultiva prácticamente en todo el territorio (Aguilar, 2012). En abril del 2014, la Financiera Nacional de Desarrollo, dependiente de la Secretaría de Hacienda y Crédito Público, emitió una ficha técnica sobre el Panorama del chile, de acuerdo con la cual el chile es el $8^{\circ}$ cultivo con mayor valor generado en la agricultura nacional, con un volumen de producción promedio de 2.2 millones de toneladas, del cual se exportan cerca de 900 mil toneladas de chiles frescos, secos y en preparaciones.

El chile se produce todo el año, sin embargo, el volumen de producción baja entre abril y junio. Así mismo destaca que todas las entidades del país lo cultivan, aunque la producción se concentra en Chihuahua, Sinaloa, Zacatecas, San Luis Potosí y Michoacán, que en su conjunto participaron con el $67.1 \%$ del valor y el $72.5 \%$ del volumen generados en 2012 . Los chiles pueden clasificarse en verdes y secos, éstos últimos se someten a un proceso de deshidratación para su venta (Aguilar, 2012).

El contacto de la capsaicina con las neuronas sensoriales, da como resultado la liberación de opioides, como las endorfinas, que son sustancias que bloquean el dolor y que provocan un estado placentero. El consumo sucesivo de chile provocaría entonces una descarga mayor de estas endorfinas, de manera que el consumo de chile resultaría más placentero que el dolor que podría causar (López, 2003; Long, 2008).

El chile se relaciona también con algunos efectos medicinales: aumenta el número de calorías quemadas durante la digestión, reduce los niveles de colesterol, es un anticoagulante y se le 
PREFERENCIAS DE CONSUMIDORES DE SALSAS PICANTES GUASALZAS DE COMALCALCO, TABASCO.

asocia con cualidades antioxidantes. Tradicionalmente, se usa como infusión para el asma, la tos, el resfriado; como analgésico en casos de artritis, como antiinflamatorio; incluso tienen propiedades para combatir el cáncer de próstata (Inforural, 2010).

La cultura del consumo de chile en México, hace que se tengan percepciones o creencias diferentes acerca de sus beneficios o efectos dañinos. El Fideicomiso de Riesgo Compartido (FIRCO, 2017) destaca que el chile habanero es considerado el más picante del mundo y una sola porción de habaneros tiene 128 miligramos de potasio, es alto en vitamina $C$, tiene un alto contenido de capsaicina, antiinflamatorio natural que puede ayudar a tratar la artritis y dolores de cabeza, ayuda a regular los niveles de insulina, por lo que puede ayudar a prevenir la diabetes; y debido a sus propiedades es utilizado en gastronomía, medicina e industria química. Asimismo, el $80 \%$ de la producción de chile habanero se comercializa como fruto fresco y el $20 \%$ restante se dirige a la elaboración de salsas, pastas y deshidratados y se exporta principalmente a Estados Unidos, Japón, Corea del Sur, Italia y Alemania.

En ese sentido, se explica que de acuerdo con un estudio del Gabinete de Comunicación Estratégica, el 92\% de los mexicanos acostumbra acompañar sus alimentos con salsa, ya sea incluyéndola en la preparación del platillo o agregándola una vez que está servida en el plato o envuelta en una tortilla, y que de acuerdo con una encuesta el $81 \%$ de los entrevistados considera que la salsa, su preparación y consumo distingue a los mexicanos de otros países (FIRCO, 2017).

Todas las especies de chile son originarias del continente americano. Su nombre científico Capsicum proviene del griego kapsakes o cápsula. Su nombre común proviene del náhuatl chili. Son plantas herbáceas o arbustivas pequeñas de flores blancas o rosadas polinizadas por insectos como abejas, abejorros y pulgones. El nivel de picante puede variar de una planta a otra, debido a las condiciones medioambientales y del suelo en que se encuentra la planta. Los más picantes, en orden decreciente son el chile habanero, chiltepín, piquín, de árbol, serrano, jalapeño. En Tabasco, se cultiva el jalapeño, serrano y habanero (Inforural, 2010).

El consumo de este producto es tan recurrente en México, debido a que produce cierto placer al comer, pues liberan dopaminas que son hormonas o sustancias que ayudan al disfrute (INAH, 2013). En mayo de 2017, la dependencia del Gobierno Federal denominada FIRCO, publicó un 
PREFERENCIAS DE CONSUMIDORES DE SALSAS PICANTES GUASALZAS DE COMALCALCO, TABASCO.

en su portal de internet datos sobre la denominación de origen del chile habanero, la cual certifica su autenticidad y que poseen, desde el año 2010, las entidades que forman la Península de Yucatán, que son: Yucatán, Quintana Roo y Campeche; aunque se produce también en otros estados como Baja California Sur, San Luis Potosí, Sonora y Tabasco.

Salsas Picantes Guasalzas es una microempresa del municipio de Comalcalco, Tabasco dedicada a la elaboración y comercialización de salsas picantes estilo casero. Cuenta con seis años de haber sido fundada y actualmente distribuye sus productos en ciudades y zonas turísticas del estado de Tabasco. Su participación en el mercado es muy pequeña y no es reconocida por el público consumidor por lo que aún no se comercializa en gran escala.

La empresa ha logrado captar clientes, específicamente de negocios relacionados con los alimentos en lugares, como: Comalcalco, Paraíso y Villahermosa, en Tabasco, y en algunas ciudades de otros estados del país, como San Cristóbal en Chiapas, en el Puerto de Veracruz, en la ciudad de Puebla, Ciudad de México, Toluca en el Estado de México, Cancún y Playa del Carmen en Quintana Roo.

Actualmente, las variedades de Salsas Guasalzas que se comercializan son: la salsa de habanero soasado, de habanero maduro, habanero verde, salsa vinagreta de habanero ahumado y especias, salsa vinagreta de chiles secos y chocolate, salsa de chile habanero verde cremosa, Salsa de chile habanero maduro cremosa, Salsa de chile de árbol cremosa y Salsa de chile de árbol cítrica.

En un principio las salsas Guasalzas se comercializaban en envases de plástico, fue para el año 2018 que se introduce el envase de vidrio. El precio de mayoreo es de $\$ 30.00$ la unidad, siendo el costo de la caja con 24 envases de \$720.00; y el precio de menudeo sugerido de $\$ 35.00$ a $\$ 55.00 \mathrm{c} / \mathrm{u}$.

\section{MATERIAL Y METÓDO}

El presente estudio se llevó a cabo en el municipio de Comalcalco, Tabasco, localizado entre la región del rio Grijalva y en la subregión de la Chontalpa; también se le llama popularmente, "La Perla de la Chontalpa", se encuentra ubicado en la región noroeste de Tabasco. De acuerdo con los datos del (Ayuntamiento Constitucional de Comalcalco, 2016) (ver tabla 1), la población total del municipio en 2010 fue de 192,802 personas, la cual representó el 8.6\% de la población 
PREFERENCIAS DE CONSUMIDORES DE SALSAS PICANTES GUASALZAS DE COMALCALCO, TABASCO.

total en el estado. Se reportó, para el mismo año, que había en el municipio 46,466 hogares (8.3\% del total de hogares en la entidad), de los cuales 9,629 (20.7\% de los hogares del municipio) estaban encabezados por jefas de familia. En cuanto al tamaño promedio de los hogares, en el municipio fue de 4.1 integrantes, mientras que en el estado el tamaño promedio de los hogares fue de cuatro integrantes.

Tabla 1

Características sociodemográficas del municipio de Comalcalco, Tabasco, 2010

\begin{tabular}{|c|c|c|}
\hline Indicador & Comalcalco & Tabasco \\
\hline Población total. & 192,802 & $2,238,603$ \\
\hline $\begin{array}{l}\text { Total de hogares y viviendas particulares } \\
\text { habitadas. }\end{array}$ & 46,466 & 559,114 \\
\hline Tamaño promedio de los hogares (personas). & 4.1 & 4 \\
\hline Hogares con jefatura femenina. & 9,629 & 129,184 \\
\hline $\begin{array}{l}\text { Grado promedio de escolaridad de la población } \\
\text { de } 15 \text { o más años. }\end{array}$ & 8 & 8.6 \\
\hline $\begin{array}{l}\text { Total de escuelas en educación básica y media } \\
\text { superior. }\end{array}$ & 388 & 5,208 \\
\hline Personal médico (personas). & 295 & 5,308 \\
\hline Unidades médicas. & 44 & 647 \\
\hline $\begin{array}{l}\text { Número promedio de carencias para la población } \\
\text { en situación de pobreza. }\end{array}$ & 3.1 & 2.7 \\
\hline $\begin{array}{l}\text { Número promedio de carencias para la población } \\
\text { en situación de pobreza extrema. }\end{array}$ & 3.9 & 3.7 \\
\hline
\end{tabular}

Fuente: Plan de Desarrollo Municipal 2016-2018, tomado del INEGI Y CONEVAL 2010.

El municipio de Comalcalco, está integrado por dos ciudades, tres villas, dos poblados, 90 rancherías y 30 ejidos; en el municipio se han ubicado 12 centros de desarrollo regional (Plan Municipal de Desarrollo 2016 - 2018).

Los encuestados fueron directamente los clientes que han consumido salsas Guasalzas, en cuanto a la edad de las personas osciló entre 15 a 60 años y de todos los niveles socioeconómicos. 
PREFERENCIAS DE CONSUMIDORES DE SALSAS PICANTES GUASALZAS DE COMALCALCO, TABASCO.

Para nuestro estudio de fuentes primarias de información se contó con 80 consumidores de salsas Guasalzas, presentaron la característica de haber probado o consumido en una o más ocasiones el producto. A las citadas personas, se les aplicó una encuesta para conocer sus preferencias en el consumo de salsas y su opinión sobre los productos de la marca; de igual manera se obtuvo información primaria de parte del propietario de la empresa Guasalzas, de los degustadores a quienes se aplicó la técnica de observación participativa y adicionalmente de los expertos a quienes se aplicaron entrevistas.

Mediante en el enfoque cuantitativo, los instrumentos de recolección de información básicamente se centran alrededor de la entrevista estructurada y la observación directa o participativa que son instrumentos y técnicas usados más frecuentemente (Martínez, 2004).

\section{Descripción del instrumento de investigación}

Se aplicó una encuesta con duración aproximada de 5 minutos; el cuestionario consistió en tres preguntas estadísticas y once relativas al tema. En la segunda parte se realizaron preguntas específicas con respecto al consumo de salsa para acompañar sus alimentos y con respecto a la variedad de chile que preferían. Unas preguntas se centraron en lo más importante para el consumidor al momento de comprar una salsa, cuál de las marcas de salsas locales le eran más conocidas, se cuestionó también acerca de sus conocimientos sobre la marca Guasalzas y se les solicitó calificar el servicio brindado por el personal de la empresa. Con respecto a la distribución, se cuestionó sobre los lugares donde les gustaría encontrar el producto y si consideraban adecuado el tamaño del envase de $150 \mathrm{ml}$. Finalmente, se interrogó sobre su disposición para comprar el producto y cuánto estarían dispuestos a pagar por una salsa de 150 ml. Asimismo, se solicitó opiniones sobre a través de cuál medio le gustaría recibir información sobre los productos Guasalzas.

\section{Características de la aplicación de las encuestas}

La recolección de datos se realizó en el campo, mediante encuesta personal apoyada con cuestionario administrado a una muestra por conveniencia de 80 personas que habían adquirido salsas Guasalzas para su consumo. También, se obtuvo información de la empresa mediante una entrevista al propietario de la misma y documentación que aportó para la investigación. 
PREFERENCIAS DE CONSUMIDORES DE SALSAS PICANTES GUASALZAS DE COMALCALCO, TABASCO.

Se complementó la observación durante el desarrollo de la investigación, al realizar pruebas piloto de las encuestas ya que se obsequiaron degustaciones de las salsas a clientes de la empresa, amistades y familiares; un aspecto interesante fue que varios encuestados pedían a comprar las salsas que les gustaban después de probarlas. También, se realizaron entrevistas a personas dedicadas a la gastronomía, con amplia experiencia en el área.

\section{Análisis estadístico}

Con las respuestas de cada entrevistado mediante la degustación, (ver figura 1), acerca de la distribución por género, donde el total de la muestra es el 61\% que corresponde a mujeres y $39 \%$ hombres. Si consideramos que la muestra fue por conveniencia para las personas que habían adquirido o consumido las salsas, se observa que las mujeres fueron quienes más mostraron interés, lo cual resulta además congruente con el conocido hecho de que las mujeres son principalmente quienes compran despensa o los bienes de consumo para el hogar.

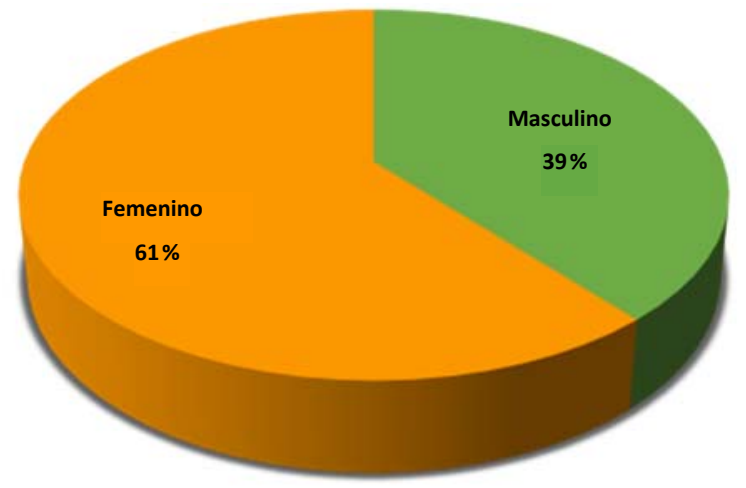

Figura 1. Distribución por género.

Fuente: Elaboración propia, con datos de la investigación.

\section{RESULTADOS Y DISCUSIÓN}

Encuesta se aplicó a una muestra por conveniencia, seleccionando a personas que han consumido las salsas Guasalzas, ya sea porque la compraron ellos directamente o algún familiar; por lo que el $\mathbf{1 0 0 \%}$ de las personas que participaron en la encuesta consumen salsas para acompañar sus alimentos. 
PREFERENCIAS DE CONSUMIDORES DE SALSAS PICANTES GUASALZAS DE COMALCALCO, TABASCO.

Como se aprecia en la figura 2, acerca de la variante o tipo de chile que más se consume, hubo diferencias significativas entre las clases de respuestas; el "habanero" fue el más consumido (46\%), seguido de "amashito" (23\%), de "jalapeño" (13\%), "chipotle" (9\%), "serrano" (9\%) y "piquín" (1\%). Aguilar (2012) señala que México es el país con la mayor diversidad de capsicum annuum, donde se cultivan en todo el territorio la producción se concentra en Chihuahua, Sinaloa, Zacatecas, San Luis Potosí y Michoacán, que en su conjunto participaron con el 67.1\% del valor y el $72.5 \%$ del volumen generados en 2012.

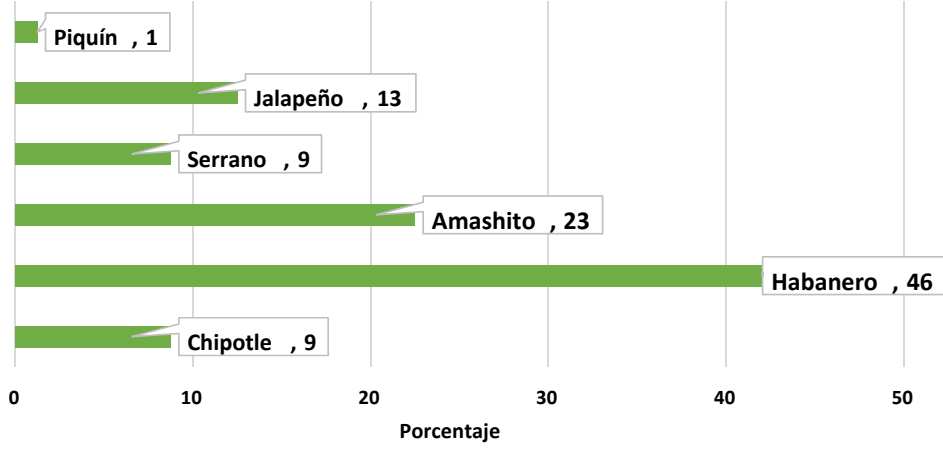

Figura 2. Variedad de chile que prefieren.

Fuente: Elaboración propia, con datos de la investigación.

Entre las razones de consumo se destaca que el sabor es la característica considerada más importante por los consumidores, preferida por el 54\% de ellos y en segundo lugar la calidad, con 31\%, (ver figura 3). Por ello, el chile es uno de los ingredientes esenciales en la cocina según (Pérez, 2014). Las preferencias de consumo parecen estar relacionadas con las percepciones del contenido de capsaicinoides, flavonoides y fenoles, entre otros compuestos, como se detectó en la evaluación de la composición de chiles regionales de Yucatán (Cázares et al., 2005), de Puebla (Morán et al., 2008).

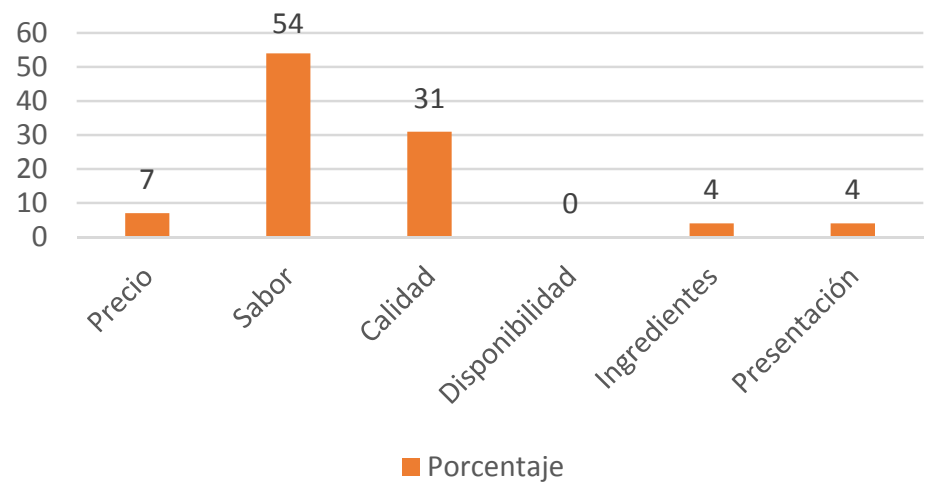

Figura 3. Características más valoradas al comprar una salsa.

Fuente: Elaboración propia, con datos de la investigación. 
PREFERENCIAS DE CONSUMIDORES DE SALSAS PICANTES GUASALZAS DE COMALCALCO, TABASCO.

Esto indica que, no obstante, la presencia de los chiles ampliamente comercializados como "Jalapeño" y "Serrano", las variantes locales tienen su nicho de mercado, aunque no con altos volúmenes. Esto es parte de los factores que contribuyen para que no se pierda la diversidad de tipos locales de chile en las diferentes regiones de México, lo que favorece su conservación basada en la utilización o consumo. Este planteamiento, es apoyado por los trabajos de (Aguilar et al., 2010) y (Lesur, 2006), en relación con la idea de que la mayor diversidad de Capsicum en México sólo es conocida y utilizada a nivel regional o local.

Los resultados de la encuesta de la presente investigación coinciden con (Pérez, 2014), quien afirma que entre las razones de consumo se destaca el sabor con un $69.9 \%$ y el picor 20\%. Por ello, el chile es uno de los ingredientes esenciales en la cocina oaxaqueña según (Pérez, 2014).

En este trabajo se encontraron datos muy significativos ya que se relacionaron los resultados de diferentes preguntas. Por ejemplo se relacionó las preferencias de los consumidores con respecto al chile local que más se consume con las razones y formas de consumo, así como con el chile local que más se adquiere o compran. Esto es de relevancia cultural y conveniente para la conservación de los acervos genéticos de los chiles locales, porque indica, hasta cierto punto, una convicción en las preferencias del consumo de los chile regionales como parte de la reafirmación cultural (Pérez, 2004; Long, 2008). En este caso, para la preparación de platillos regionales. Además, estos hechos están vinculados con aspectos de mercado regional o nacional; si bien, el consumo y preferencia se relacionan con aspectos socioculturales locales, las influencias del mercado son determinantes en los cambios de patrones de consumo que en ocasiones no es a favor de alimentos tradicionales o de alto valor nutritivo (G. Pelto y P. Pelto, 1983).

\section{CONCLUSIONES}

En conclusión podemos señalar que la empresa Salsas Picantes Guasalzas, tiene amplias posibilidades de comercializar con éxito sus productos, debido a que el $100 \%$ de los encuestados son susceptibles de consumirlos. También es pertinente indicar la empresa fábrica salsas a base de los chiles habanero y amashito que son los más consumidos por la población encuestada. Por otra parte, las Salsas Guasalzas cuentan con una buena percepción de sabor y calidad, características que son muy apreciadas por los consumidores de salsas. 
PREFERENCIAS DE CONSUMIDORES DE SALSAS PICANTES GUASALZAS DE COMALCALCO, TABASCO.

El precio es aceptable y se obtuvo respuesta favorable durante las degustaciones realizadas en las pruebas piloto, en las que los participantes pedían a comprar las salsas y varios de ellos continúan comprándolas; siempre y cuando estén disponibles y a la vista, ya que si las ven preguntan por los productos. Cuando las personas preguntan, el vendedor tiene la oportunidad de explicar sobre las presentaciones y los usos sugeridos, también se abre la posibilidad de que compren y posteriormente regresan por más producto o que inclusive, puedan adquirirlas para obsequiar a sus conocidos.

El consumo de salsas es muy intenso, tanto por el volumen que se consume, como por el grado de picor de los chiles que se prefieren. En las encuestas, el resultado fue que el chile habanero es el preferido de los consumidores en esta zona geográfica, seguido por el amashito. Los datos recuperados en la investigación representan una ventaja para Guasalzas, ya que el chile habanero es uno de los ingredientes principales que se utilizan para la elaboración de las salsas de esta marca.

Por lo que respecta a la plaza, los principales lugares donde se esperaría encontrar las Salsas Guasalzas son los supermercados y tiendas de conveniencia; sin embargo, en estos momentos, dada la escasez de capital y el nivel de producción, el empresario aún no considera viable entrar en estos establecimientos. Lo anterior, debido a la experiencia de otros empresarios ya que se tiene conocimiento de que se presentan mermas, pérdida de producto y competencia desleal por parte de las grandes empresas del mismo ramo, lo cual no está en condiciones de absorber en estos momentos.

Por último, los medios que los encuestados manifestaron preferir para que se les haga llegar información de los productos son en orden de preferencia redes sociales, televisión y en menor porcentaje folletos y radio, lo cual resulta congruente con el desarrollo de la tecnología y la proliferación del uso de las redes sociales y el internet en general como medios de comunicación para dar a conocer productos e impulsar las ventas. A través de dichos medios se tienen muchas ventajas, así como la posibilidad de mostrar información e imágenes de los productos, videos sobre los usos, testimonios, resaltar sus atributos y mostrar sus beneficios.

Tomando en cuenta los resultados de la investigación, así como la situación financiera actual de la empresa, se propone una serie de estrategias para incrementar las ventas de productos Guasalzas y con ello la rentabilidad de la empresa. 
PREFERENCIAS DE CONSUMIDORES DE SALSAS PICANTES GUASALZAS DE COMALCALCO, TABASCO.

\section{PROPUESTA}

El presente documento, propone estrategias de comercialización para dar a conocer sus productos a un mayor número de consumidores y consecuentemente incrementar sus ventas.

- Proponer envases más grandes para venta a restaurantes.

- Estudiar las marcas de la competencia y sus características para determinar elementos posicionables o características diferenciadoras.

- Creación de una página web para venta y promoción y creación de un muro de Facebook en donde puedan enviar publicidad y mensajes directos a los consumidores por lo que es importante que se atiendan con eficiencia y prontitud los mensajes.

- Fortalecer el posicionamiento y difusión para que las Salsas Guasalzas sean más conocidas.

- Ocasionalmente realizar pruebas de degustación de las presentaciones de salsas menos conocidas, en el mismo local de la empresa para impulsar las ventas.

Las propuestas están clasificadas de acuerdo a la estrategia de marketing (producto, precio, plaza y promoción) descrita por (Ferrer, Hartline y Michael, 2012).

\section{REFERENCIAS BIBLIOGRÁFICAS}

Aguilar, V. (2012). Cultivo del chile en México. Revista futotecnica mexicana, 35(4), 264-264. Recuperado de http://www.scielo.org.mx/scielo.php?script=sci_arttext\&pid=S018773802012000400001.

Aguilar, V., Corona, T., López, P., Latournerie, L., Ramírez, M., Villalón, H. y Aguilar, J. (2010). Los chiles de México y su distribución. Montecillo, Texcoco, Estado de México: SINAREFI, CP, INIFAP, IT-CONKAL, UANL y UAN

Ayuntamiento Constitucional de Comalcalco. (2016). Plan de Desarrollo Municipal, Comalcalco 2016-2018. http://www.comalcalco.gob.mx/publica/pdf/programacion/pmd_2016_2018.pdf 
PREFERENCIAS DE CONSUMIDORES DE SALSAS PICANTES GUASALZAS DE COMALCALCO, TABASCO.

Cázares, E., Ramírez, P., Castillo, F., Soto, M., Rodriguez, M. y Chávez, J. (2005). Capsaicinoides y preferencias de uso en diferentes morfotipos de chile (capsicum annuum L.) del centro- oriente de yucatan. Agrociencia, 39(1), 227-238.

Ferrel, O. y Hartline, M. (2012). Estrategia de Marketing. México: CENGAGE Learning.

FIRCO. (2017). Chile habanero, con denominación de origen. Recuperado de https://www.gob.mx/firco/articulos/chile-habanero-con-denominacion-de-origen?idiom=es

INAH. (2013). Chiles y salsas en México. Un sabor a identidad. Recuperado de https://www.inah.gob.mx/reportajes/597-chiles-y-salsas-en-mexico-un-sabor-a-identidad.

Inforural. (2010). México: primer lugar mundial en producción de chile verde y sexto en la de chile seco. Recuperado de https://www.inforural.com.mx/mexico-primer-lugar-mundialen-produccion-de-chile-verde-y-sexto-en-la-de-chile-seco/

Lesur , L. (2006). Manual del cultivo del chile. México, D.F: Trillas.

Long, J. (2008). Tecnología alimentaria prehispánica. Estudios de cultura Náhuatl, 39, 127-136. Recuperado de http://www.revistas.unam.mx/index.php/ecn/article/view/15291/14537

López, G. (2003). Chilli: la especia del nuevo mundo. Ciencias, 69, 66-75. Recuperado de https://www.researchgate.net/publication/232670090_Chilli_La_Especia_del_Nuevo_Mu ndo_Chilli_The_Spice_of_the_New_World.

Martínez, M. (2004). Ciencia y arte en la metodología cualitativa. México: Trillas.

Morán, S., Aguilar, V., Corona, T., Castillo, F., Soto, R. y San Miguel, R. (2008). Capsaicinoides en chiles nativos de puebla, México. Agrociencia, 42(7), 807-816.

Pelto, G. y Pelto, P. (1983). Diet and delocalization: dietary change since 1750 . The Journal of Interdisciplinary History, 14(2), 507-528. DOI 10.2307/203719

Pérez, E. (2004). Recetario mixe de Oaxaca. México, D.F.: CONACULTA. 